\title{
Parallel processing techniques in EMP propagation using 3D Finite-Difference Time-Domain (FDTD) method
}

\author{
W. J. Buchanan \& N. K. Gupta \\ Department of Electrical, Electronic and Computer Engineering, Napier University, 219 Colinton Road, Edinburgh \\ EHI4 $1 D J, U K$
}

(Received 1 June 1993; revised version received 4 October 1993; accepted 15 October 1993)

\begin{abstract}
The authors have applied the 3D FDTD technique to simulate the propagation of electrical signals on a microstrip antenna using a four-transputer array connected to a 386-based PC. This relates to work currently being carried out into the propagation of very high speed digital pulses in printed circuit boards in high bandwidth systems. Methods have been devised by the authors which reduce the amount of stored data and improve run-time. These methods include subgridding, shielded volume and concurrent processing of the problem. Results have been obtained and are presented in this paper to show improved accuracy and decreased run-time. An analysis of run-time to the number of transputers in an array for optimum operation has been presented. A trade-off between intertransputer communication of segment boundary conditions and the size of each segment has also been discussed in the paper.
\end{abstract}

Key words: parallel processing, 3D FDTD method, EMP propagation, microstrip antenna, Maxwell's equations.

\section{INTRODUCTION}

Many electrical engineering problems can be easily formulated and expressed using Maxwell's equations. These equations define exactly the propagation of all electrical signals but their exact solutions are complex and tedious. There are, however, some approximate methods that are easier and more widely used. Three well-established methods for approximate solutions are:

- Finite-difference method

- Variations method

- Finite-element method

The next generation of computers will bring a revolution by maximising the use of parallel processing in computation. With this tochnology full timedomain solutions will be possible and frequency information can be extracted using Fast Fourier Transform techniques. The most relevant method for parallel systems is the Finite-Difference Time-Domain (FDTD) method.

Maxwell's equations define the propagation of an electromagnetic wave, such as radio, microwave, infrared, optical, ultraviolet, etc. They define the relationship between the electric field and the magnetic field and are given below:

$$
\begin{aligned}
\mu \frac{\delta \mathbf{H}}{\delta t} & =-\nabla \times \mathbf{E} \\
\epsilon \frac{\delta \mathbf{E}}{\delta t}+\mathbf{J} & =\nabla \times \mathbf{H} \\
\nabla \cdot \mathbf{E} & =\rho \\
\nabla \cdot \mathbf{H} & =0
\end{aligned}
$$

The first two equations show that a change in electric field (E) produces a magnetic field $(\mathbf{H})$ and vice-versa. The third equation shows that the electric field (E) is related to electric charge $(\rho)$. The last equation shows that the magnetic field relates to magnetic charge (although it does not exist).

The FDTD method is a novel method in that it provides for a direct solution to Maxwell's equations without much complexity. Also this method takes all fields (electric and magnetic) in a three-dimensional model into account; other empirical analytical methods do not. 


\section{FINITE-DIFFERENCE TIME-DOMAIN (FDTD) METHOD}

With increasing power and memory storage of modern computers it is possible to simulate concepts in the time domain rather than analysing them in the frequency domain. Another change in techniques has been to convert continuous equations into discrete forms. These discrete forms are usually much easier to implement on a digital computer. A good example of the use of timedomain simulation using a discrete equation is in the FDTD method. This technique also determines the frequency response over a wide spectrum of frequencies, whereas other methods would require different models and/or techniques for different frequency spectra.

For uniform, isotropic and homogeneous media Maxwell's curl equations can be simplified as:

$$
\begin{gathered}
\mu \frac{\delta \mathbf{H}}{\delta t}=-\nabla \times \mathbf{E} \\
\epsilon \frac{\delta \mathbf{E}}{\delta t}=\nabla \times \mathbf{H}
\end{gathered}
$$

In order to find an approximate solution to this set of equations, the problem is made discrete over a finite three-dimensional computational domain with appropriate boundary conditions imposed on the source, conductors and mesh walls. Taking an example of the first equation in the $i$ direction:

$$
\mu \frac{\Delta H_{x}}{\Delta t}=\frac{\Delta E_{y}}{\Delta z}-\frac{\Delta E_{z}}{\Delta y}
$$

In order to obtain discrete approximations the central difference approximation is used on both the time and space first-order partial differentiations; this gives

$$
\mu \frac{H_{x i, j, k}^{n+1 / 2}-H_{x i, j, k}^{n-1 / 2}}{\Delta T}=\frac{E_{y i, j, k}^{n}-E_{y i, j, k-1}^{n}}{\Delta z}-\frac{E_{z i, j, k}^{n}-E_{z i, j-1, k}^{n}}{\Delta y}
$$

Rearranging gives the following:

$$
\begin{aligned}
H_{x i, j, k}^{n+1 / 2}= & H_{x i, j, k}^{n-1 / 2}+\frac{\Delta t}{\mu \Delta z}\left[E_{y i, j, k}^{n}-E_{y i, j, k-1}^{n}\right] \\
& -\frac{\Delta t}{\mu \Delta y}\left[E_{z i, j, k}^{n}-E_{z i-1, j, k}^{n}\right]
\end{aligned}
$$

The half-time steps indicate that $E$ and $H$ are alternately calculated in order to achieve central

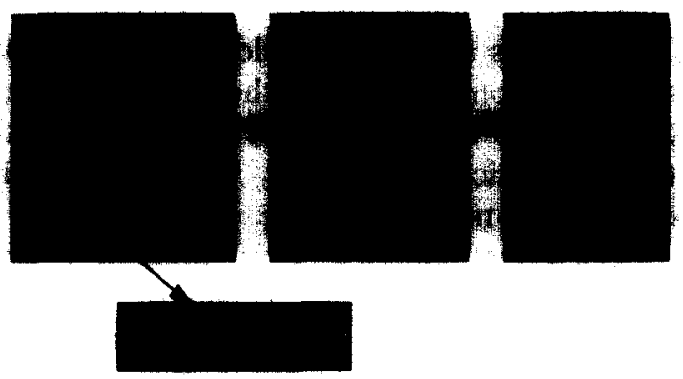

Fig. 1. FDTD method. differences for the time derivatives. In these equations, the permittivity and the permeability are set to the approximate values depending on the location of each field component. In total there are six equations similar to the one given above, which define the $E$ and $H$ fields in the $x, y$ and $z$ directions. The method is outlined in Fig. 1.

\section{Problem conception}

The structure simulated in this paper is a microstrip antenna, which is used to radiate microwave energy at a desired resonant frequency. The transient analysis of the antenna is complex, as it involves multiple reflections and is highly resonant at certain frequencies. The physical structure of the antenna consists of a substrate layer, such as Duroid (dielectric constant of $2 \cdot 2$ ), and a ground plane below this layer. A copper layer is formed by etching off a portion from the top of the substrate to give the required pattern. The diagram in Fig. 2 shows an example of a microstrip antenna with an $x y z$ grid superimposed on the structure.

To analyse this structure using the 3D FDTD method the following model considerations are to be included.

\section{(1) Dielectrics}

In air, the relative dielectric constant is $\epsilon_{\mathrm{r} 1}$; in dielectric, it is $\epsilon_{\mathrm{r} 2}$; at the interface between the air and the dielectric, the relative dielectric constant is the average of those of the air and the dielectric, i.e. $\left(\epsilon_{\mathrm{r} 1}+\epsilon_{\mathrm{r} 2}\right) / 2$.

\section{(2) Time step}

The maximum time step that may be used is limited by the stability restriction of the finite difference equations. It is given by

$$
\Delta t<\frac{1}{c}\left(\frac{1}{\Delta x^{2}}+\frac{1}{\Delta y^{2}}+\frac{1}{\Delta z^{2}}\right)^{-1 / 2}
$$

where $c$ is the speed of light $\left(3 \times 10^{8} \mathrm{~m} / \mathrm{s}\right)$ and $\Delta x, \Delta y$ and $\Delta z$ are the dimensions of the unit cell.

(3) Source

A Gaussian pulse is applied to the source. This pulse is used because its frequency spectrum is also Gaussian and will provide frequency-domain information for DC to the desired cut-off frequency by adjusting the width of the pulse. The width of the Gaussian pulse is chosen for

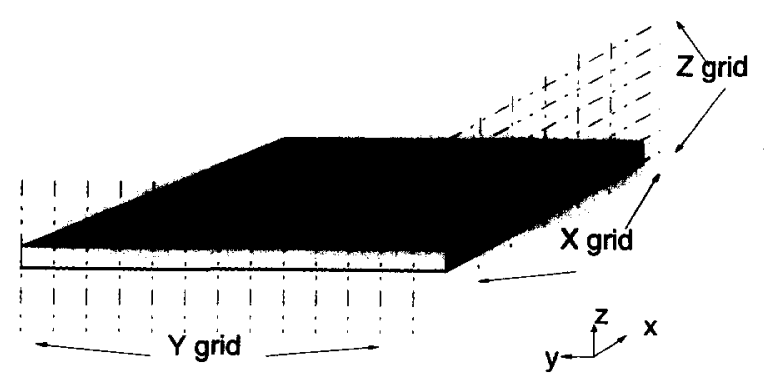

Fig. 2. Microstrip antenna with grid. 


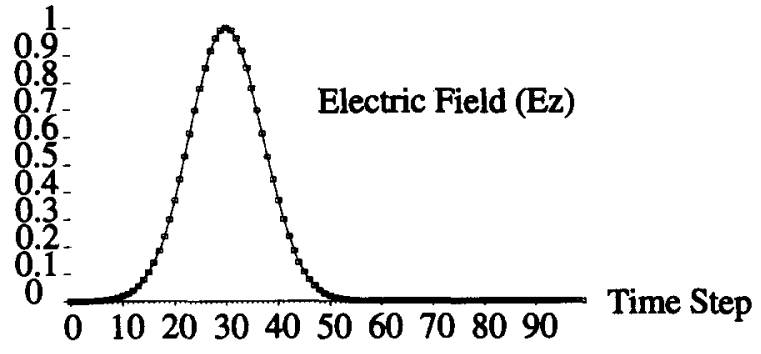

Fig. 3. Gaussian pulse.

at least 20 points per wavelength at the highest frequency represented significantly in the pulse. The electric field applied to the source has only a $z$ component and is Gaussian. The Gaussian pulse is shown in Fig. 3.

Frequency information is extracted by conducting a Fast Fourier Transform (FFT) on the time response. Several points are monitored to obtain the required response. For example, to determine the reflection coefficient of the antenna a point is chosen at the source and the reflected wave is monitored. If the radiation pattern is required the points are taken in free space.

\section{(4) Conductors}

The microstrip antenna has a conducting ground plane and a single dielectric substrate with a copper layer etched for the required shape. In the FDTD method these electrical conductors are assumed to be perfect and to have zero thickness. If this is the case then the tangential electric field components that lie on the conductors are assumed to be zero. The example in Fig. 4 shows that the $E$ field components on the conductor will be zero in the $x$ and $y$ directions.

(5) Absorbing boundary treatment

Due to memory and speed limitations of most computers there is only a finite size of a mesh cell that can be implemented. Thus an absorbing boundary must be placed around the model. There will be six boundary mesh walls. The ground plane and its tangential electric fields will be set to 0 . The tangential electric fields on the other five mesh walls must be specified so that a wave propagating against them does not reflect back. For the microstrip antenna simulated in this paper the pulses in

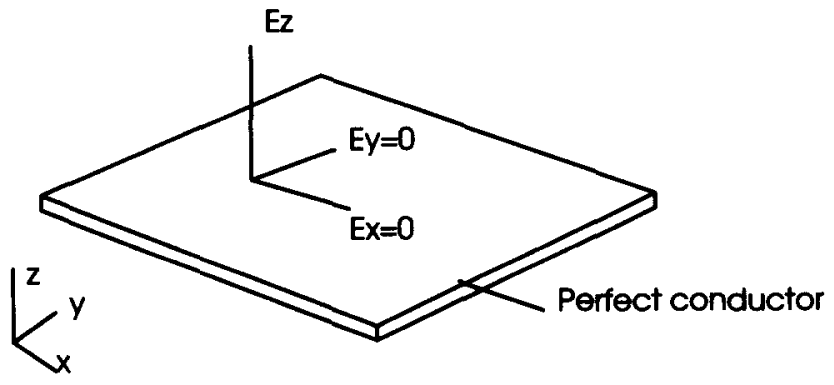

Fig. 4. Conductor treatment. the structure will be normally incident on the mesh walls. This will lead to a simple approximation for continuous absorbing boundary conditions, which is that the tangential fields on the absorbing boundaries will obey the one-dimensional wave equation in the direction normal to the mesh wall. For the normal $y$ direction wall the one-dimensional wave equation may be written as

$$
\left(\frac{\partial}{\partial y}-\frac{1}{v} \frac{\partial}{\partial t}\right) E_{\tan }=0
$$

This equation is Mur's first approximate absorbing boundary condition and this may be simply discretised to give

$$
E_{0}^{n+1}=E_{1}^{n+1}+\frac{v \Delta t-\Delta y}{v \Delta t+\Delta y}\left(E_{1}^{n+1}-E_{0}^{n}\right)
$$

where $E_{0}$ represents the tangential electric field on the mesh wall and $E_{1}$ the tangential electric field components one grid point within the mesh wall. Similar equations can be derived for the other five absorbing mesh walls. Note that this method does not take into account fringing fields which are propagating tangential to the walls; therefore the absorbing boundary is placed well away from any fringing fields. This method is well suited to microstrip problems.

\section{(6) Frequency conversion}

The FDTD method uses a time domain solution of the problem so if frequency information is required (such as the reflection coefficient) then a Fourier Transform must be conducted on the time domain solution. Since the time domain solution is in a discrete form, a Fast Fourier Transform (FFT) can be used to extract frequency data. The scattering parameters $S_{j k}$ may be obtained by simple Fourier transform of the transient waveforms as

$$
S_{j k}(\omega)=\frac{F F\left(V_{j}(t)\right)}{F F\left(V_{k}(t)\right)}
$$

For example, if the $S_{11}$ parameter is required the electric field at the input is monitored. Thus $S_{11}$ will be the reflected wave divided by the inputted wave.

\section{Parallel processing of 3D FDTD method}

Simulation of the structure on different computer systems shows the need for a maths co-processor. A maths co-processor fitted into a PC improves run-time more than ten-fold. A graph of typical simulation times for a $28 \times 50 \times 6$ grid for 50 time steps is shown in Fig. 5. The computer used is either a 386/486-based PC with or without a maths co-processor (MCo) or an HP400 workstation.

The next generation of computers will have an architecture which will support truly multiple processing systems, with different processes running off a 


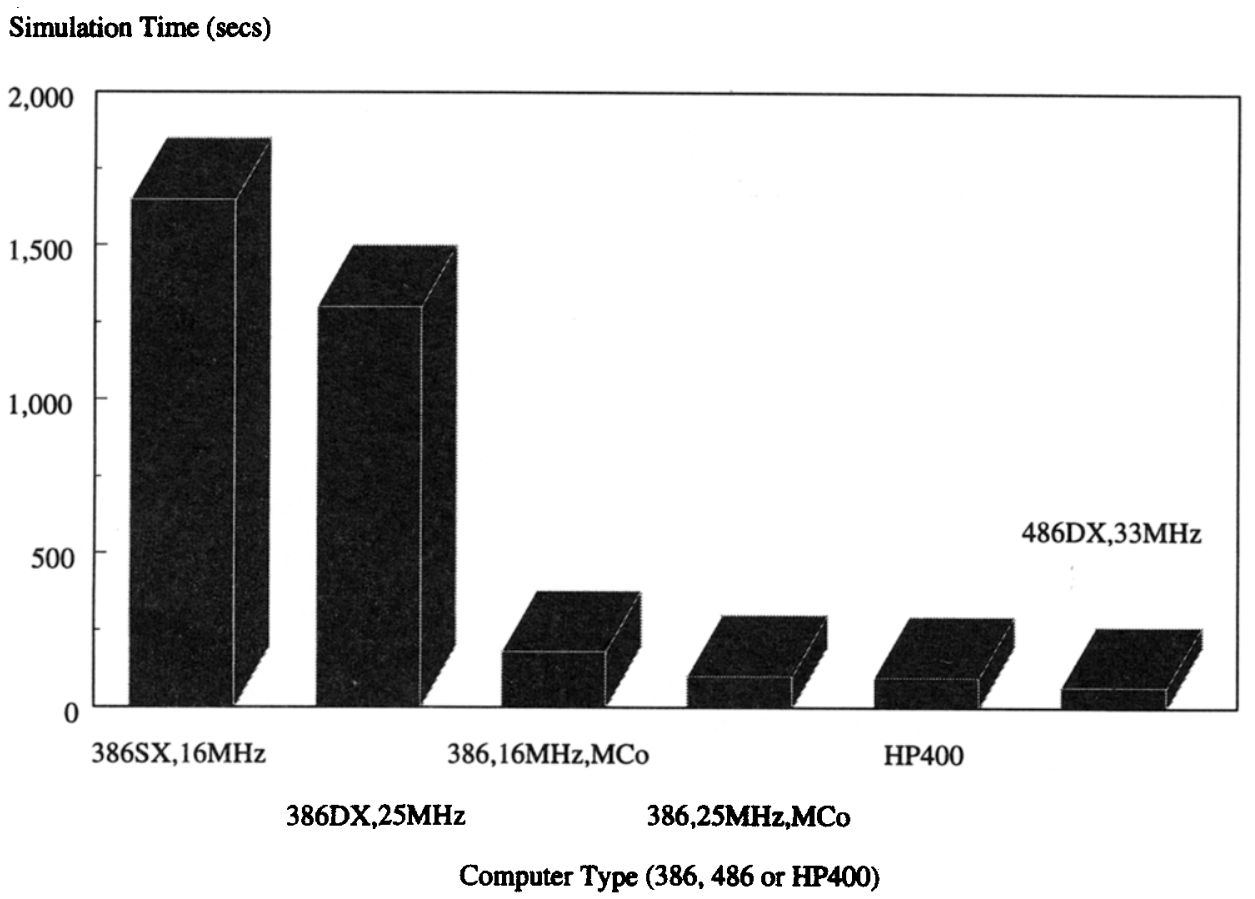

Fig. 5. Simulation time with $28 \times 50 \times 6$ grid for 50 time steps.

unique microprocessor. These systems will be able to run many processes concurrently.

Currently transputers can be fitted into a PC or workstation and can be configured to run multiple processes, even when operating under a single user operating system such as DOS. A transputer (shown in Fig. 6) can execute an application as a single process on one processor, but can be used with other transputers to form a large array in which each transputer communicates with its neighbouring transputers by means of point-to-point communication (as shown in Fig. 7). A typical transputer has a 32-bit RISC processor, onboard and local memory, full 64-bit float point processing and high speed serial link to communicate with other transputers. Each transputer is equivalent to a microcomputer and can run at $30 \mathrm{Mips}, 4 \cdot 3 \mathrm{Mflops}$. It can address up to 4 Gbytes of RAM and communicate at 5,10 or $20 \mathrm{Mbits} / \mathrm{s}$. A transputer does not have the problems of segmented memory ( $64 \mathrm{kbyte}$ chunks and maximum addressable memory of 1 Mbyte) that a PC running 8086/DOS compatible code has.

To simulate the microstrip antenna of Fig. 2, the problem must be segmented into physical domains.

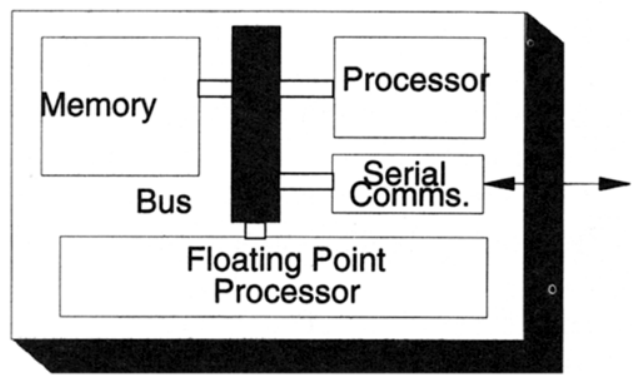

Fig. 6. A transputer.
Each transputer will operate on one of these domains, as shown in Fig. 8. An advantage of the 3D FDTD method is that the present calculations depend only on the previous time-step calculations and that no cell has to wait until the neighbouring cells have completed their calculation for input to this cell (sequential method of sweeping over a problem).

The problem with any parallel processing task is that the boundary conditions need to be transmitted after each iteration. Even if these boundary conditions are transmitted at $1,5,10$ or $20 \mathrm{Mbits} / \mathrm{s}$, the communications can have a large overhead in time. Thus, for a given size of problem, the more the number of parallel processes, the more is the time spent with the interdomain communications (transmitting boundary conditions for each domain). The actual time to compute the simulation will reduce by a factor determined by the number of transputers. The total time taken, then, will be the sum of the actual computation time and the total

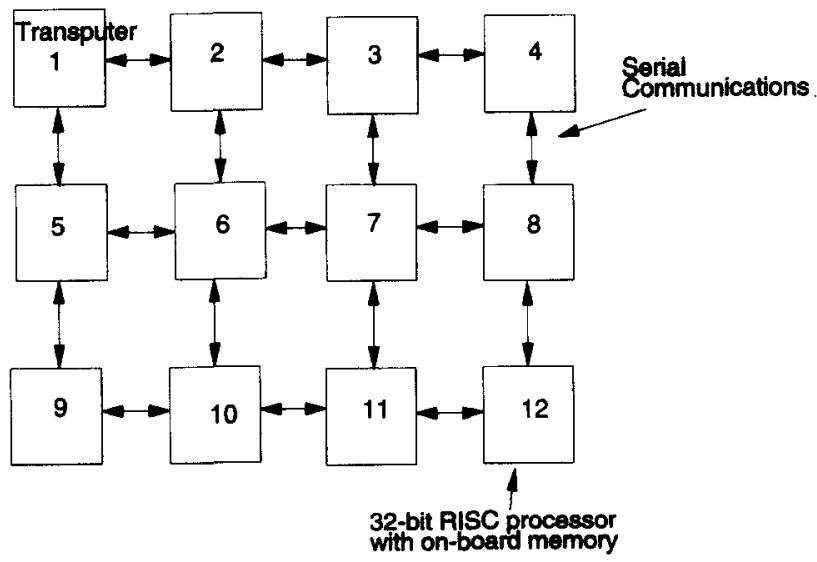

Fig. 7. Transputer array. 


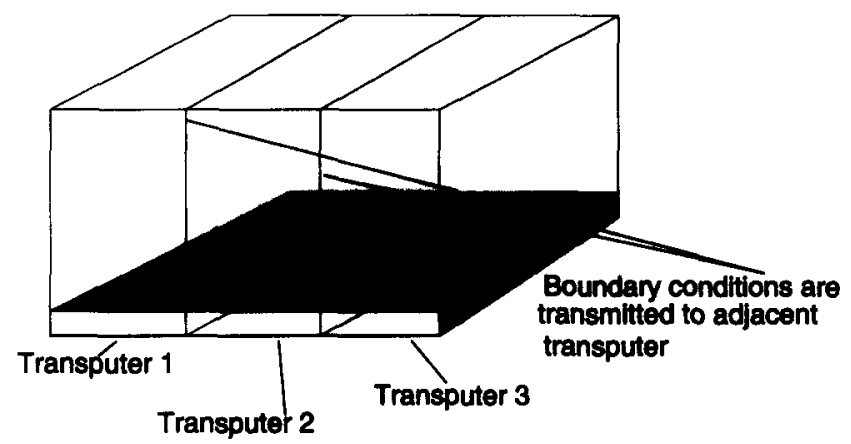

Fig. 8. Segmentation of problem with three transputers. The total number of nodes on the $x y z$ grid will be $x \times y \times z$. If a parallel processing system is used, an attempt is made to ensure that each transputer handles an equal share of nodes, so that the burden on each is relatively the same. The number of nodes which each transputer deals with will thus be

$$
\text { Number of nodes per transputer }=\frac{x \times y \times z}{N}
$$

inter-communication time. A typical simulation for a $50 \times 80 \times 12$ grid with 50 time steps is shown in Fig. 9 . These simulations are based on a $1 \mathrm{Mbits} / \mathrm{s}$ intercommunication rate, assuming four bytes per floating point value. It can be seen from the figure that the optimum number of transputers is probably about three or four. Also it can be observed that if 20 or more transputers are used, there is virtually no significant improvement in simulation time. In typical simulations, if more than 50 transputers are used, the simulation time starts to increase for increasing number of transputers. An advantage of using transputers in a PC is that a model using more than 1 Mbyte of memory can be simulated. However, there will be a limit on the maximum number of transputers that can be used to process the problem.

Another useful method in improving accuracy is to use a non-linear grid. In the absence of a discontinuity a pulse will only be effected by attenuation. Thus a fine grid can be set up around discontinuities and a less fine grid around areas which do not have discontinuities. For example, a fine grid can be set up in and just above

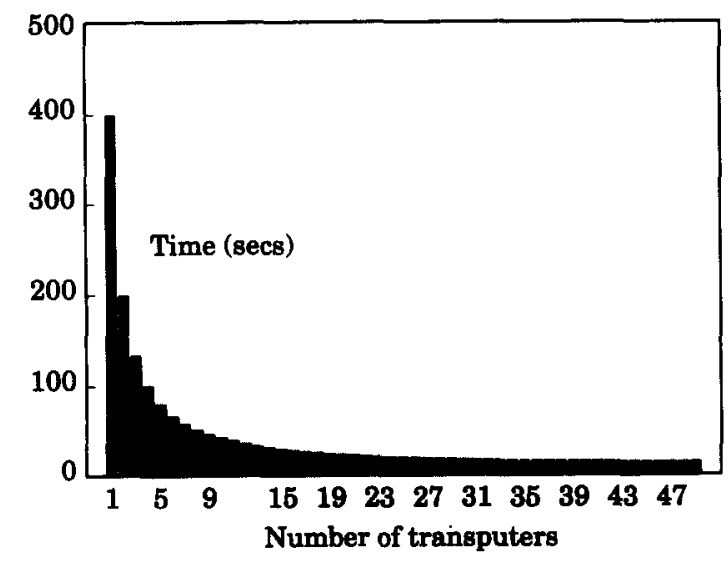

Fig. 9. Time to compute for number of transputers.

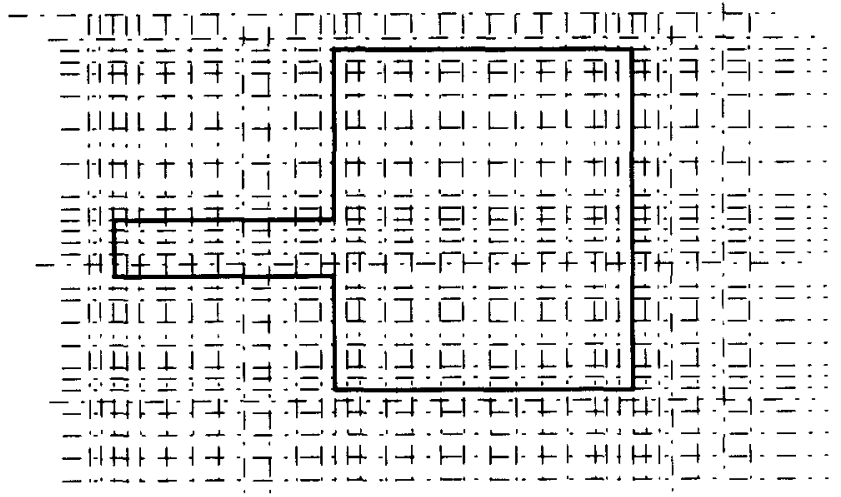

Fig. 10. Subgridding around discontinuities.

the antenna but at some distance away from the antenna a less fine grid can be used. Figure 10 shows a non-linear grid setup for the $x$ and $y$ grid.

\section{RESULTS}

The results from a simulation using a four-transputer array connected to a 386-based PC for a $50 \times 100 \times 12$ grid are shown in Figs 12-20. The simulation time was reduced to almost one-quarter of that for the equivalent sequential machine (such as PC or workstation). Thus, a relatively small amount of time was spent with intertransputer communications. The simulated antenna has a width of $12.45 \mathrm{~mm}$, a length of $16.00 \mathrm{~mm}$ and a feed width of $2.46 \mathrm{~mm}$, which is offset from the edge of the head of the antenna by $2.09 \mathrm{~mm}$. Each time step is approximately 1.25 picoseconds. The electric field plots show the field intensity in the $z$-direction and are measured just below the antenna. The return loss $\left(s_{11}\right)$ in Fig. 11 is a measure of the reflected energy at a given frequency; the less the energy returned the higher the resonated or radiated energy. This assumes that no energy is dissipated within the antenna. A return loss of $0 \mathrm{~dB}$ means that all the energy is returned to the source; at $-40 \mathrm{~dB}$ very little of the incident energy is returned. Figure 11 shows that the antenna resonates at $7.5 \mathrm{GHz}$, as expected, and that over $90 \%$ of the incident energy is

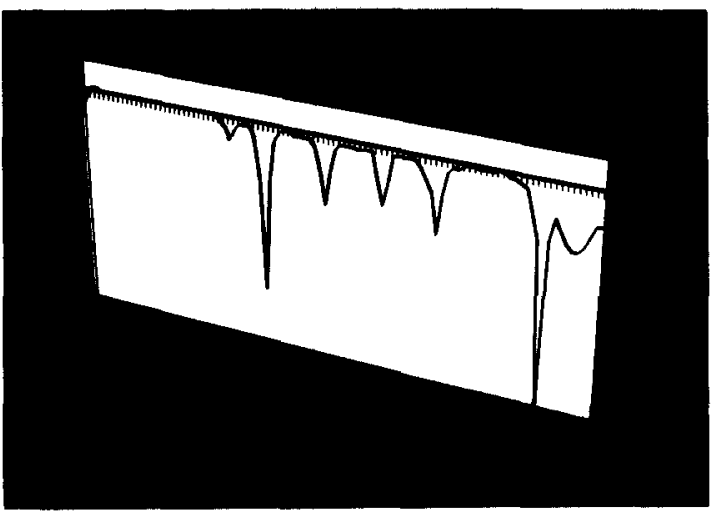

Fig. 11. Return loss (in dB) from antenna. 
3D field olot using FDTD Method

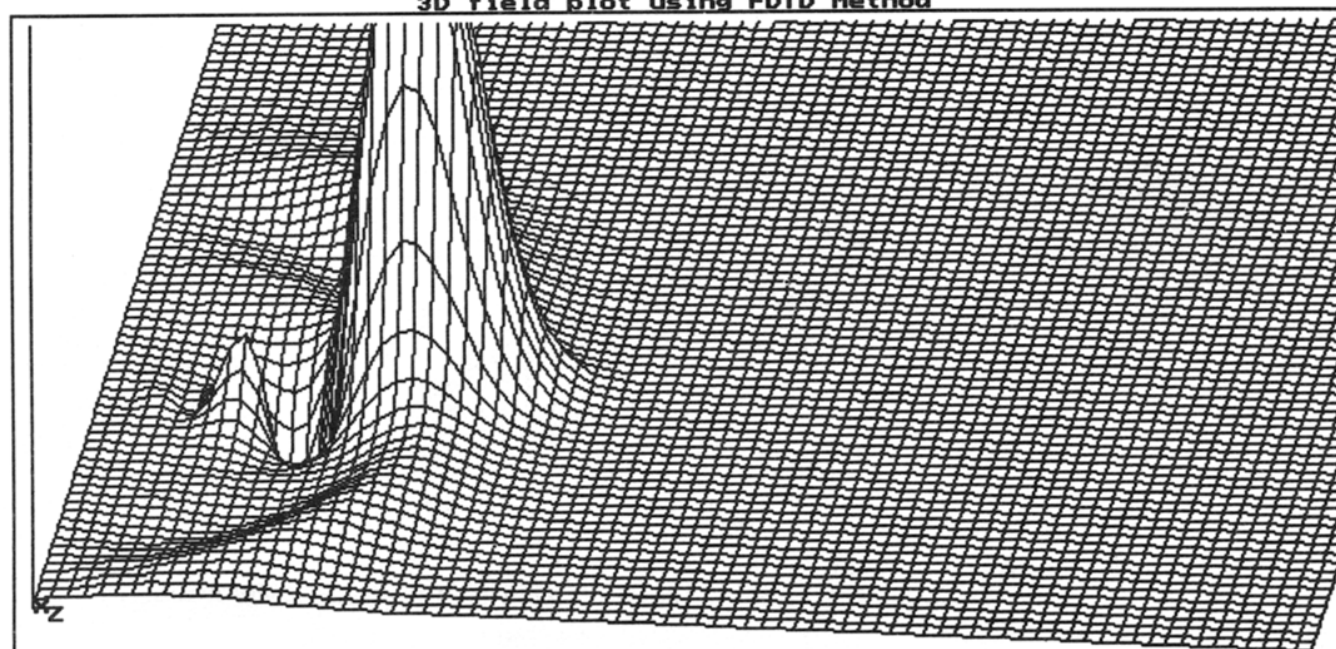

$X$ points 80 , Yoints 80 , Zslice at 1 , time step 90 (out of 5000 )

Fig. 12. Simulation after 90 time steps.

3D field plot using FDTD Method

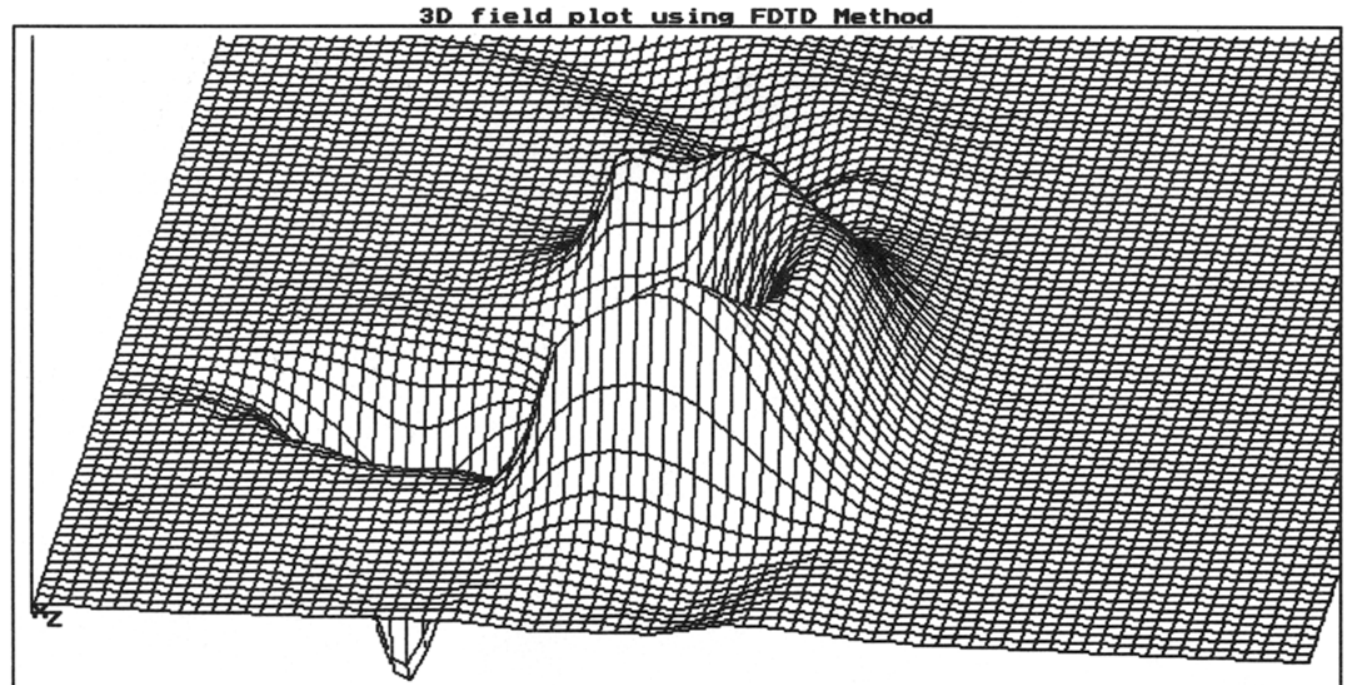

$x$ points 80 , Yooints 80 , Zslice at 1 , time step 160 (out of 5000 )

Fig. 13. Simulation after 160 time steps. 


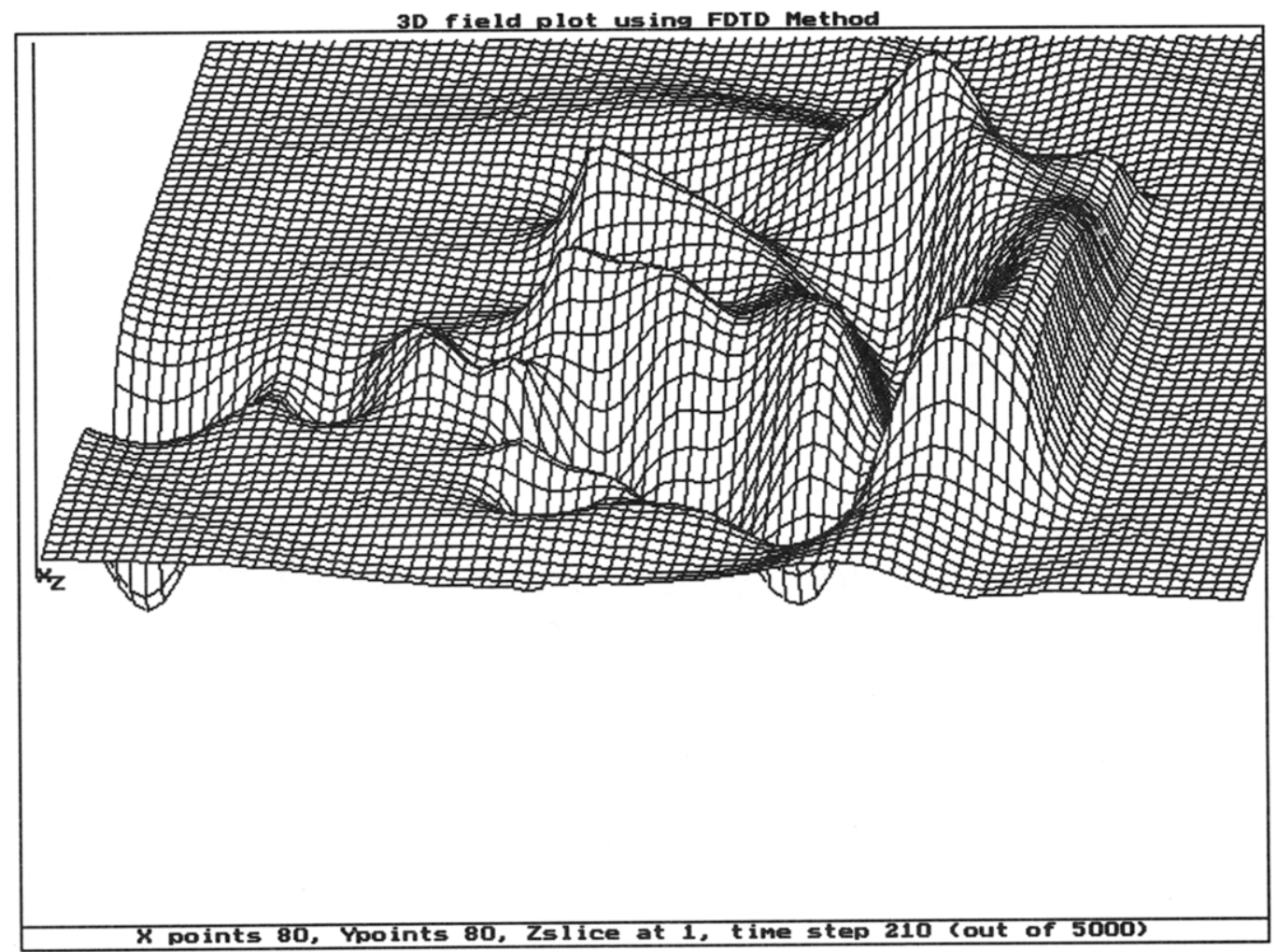

Fig. 14. Simulation after 210 time steps.

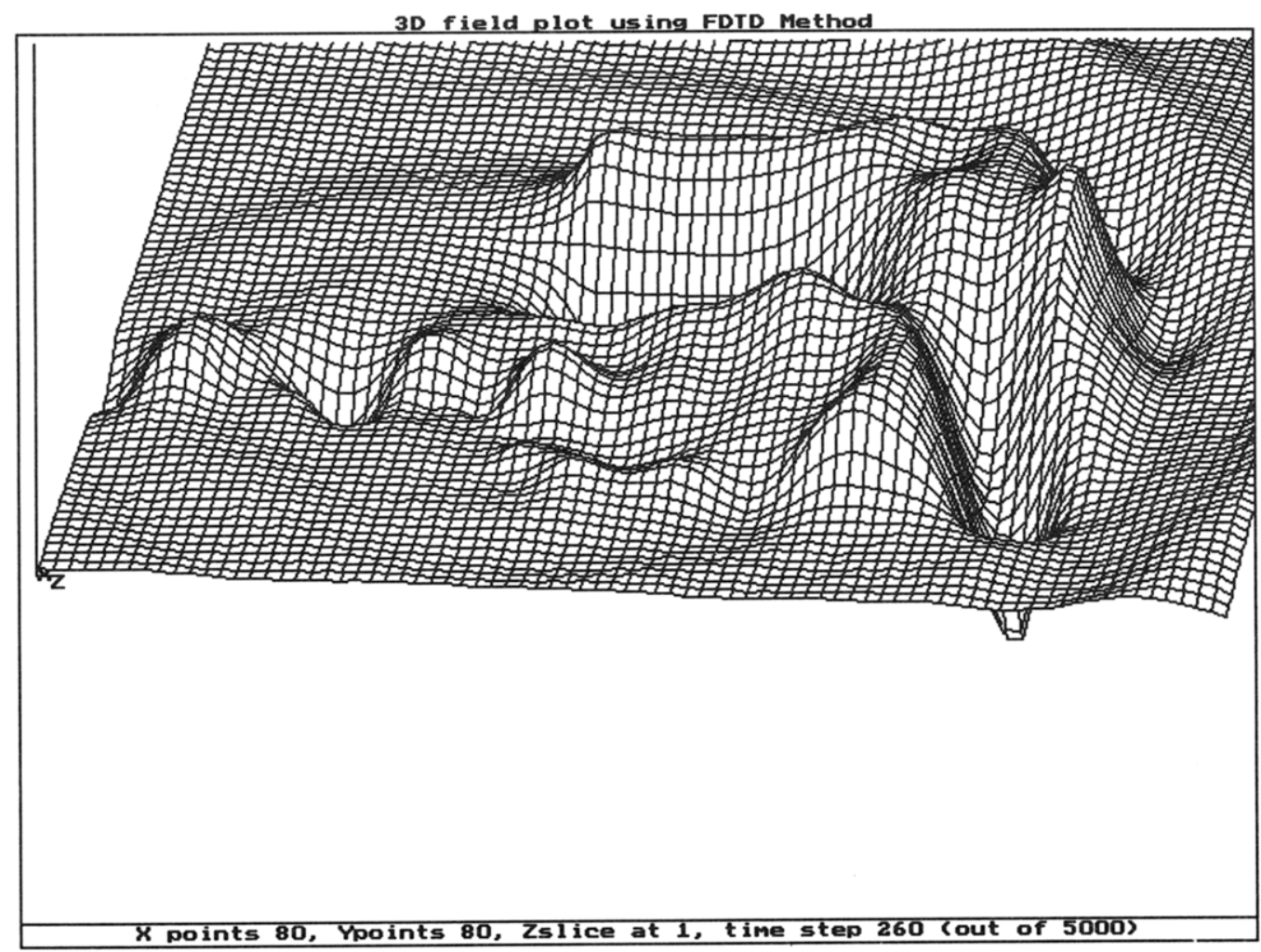

Fig. 15. Simulation after 260 time steps. 


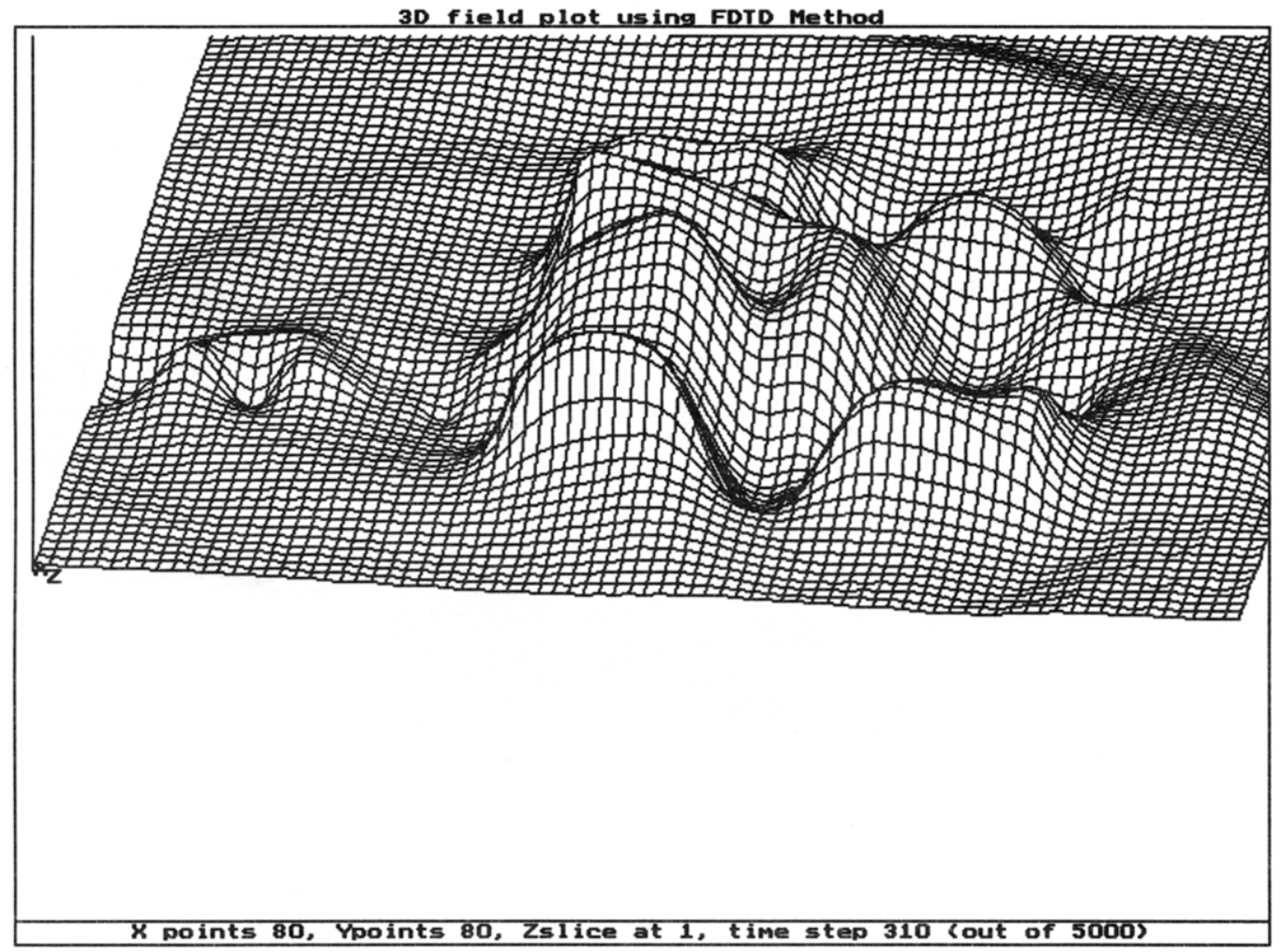

Fig. 16. Simulation after 310 time steps.

radiated at and around the frequencies of $7 \cdot 5,10,12$ and $18 \mathrm{GHz}$.

The simulated model assumes a match between the source and the antenna and an absorbing boundary around the outer walls of the problem. These values will not be totally accurate since the FDTD method used does not take into account conduction or dielectric losses. In Fig. 12, the pulse has entered the structure and

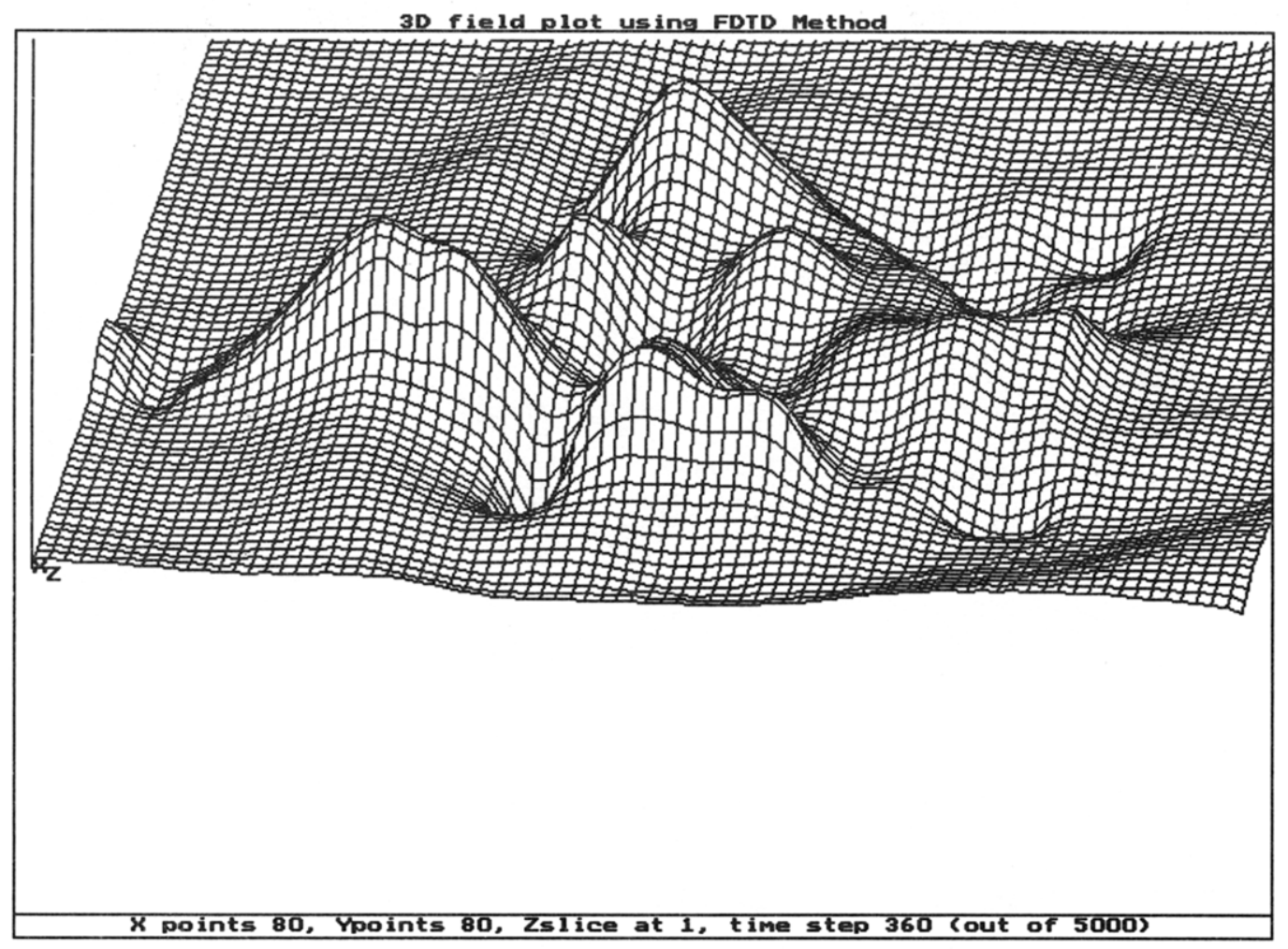

Fig. 17. Simulation after 360 time steps. 


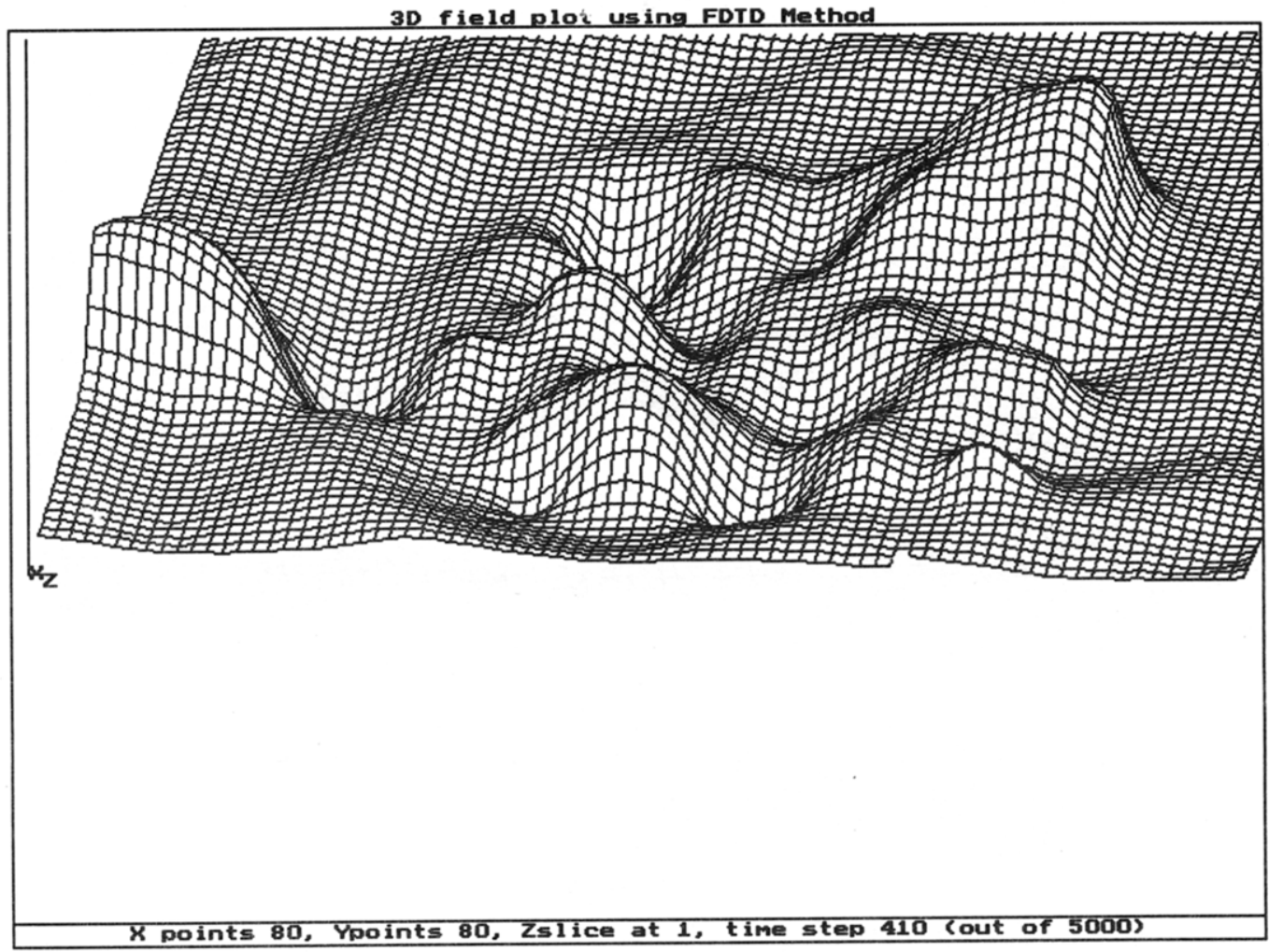

Fig. 18. Simulation after 410 time steps.

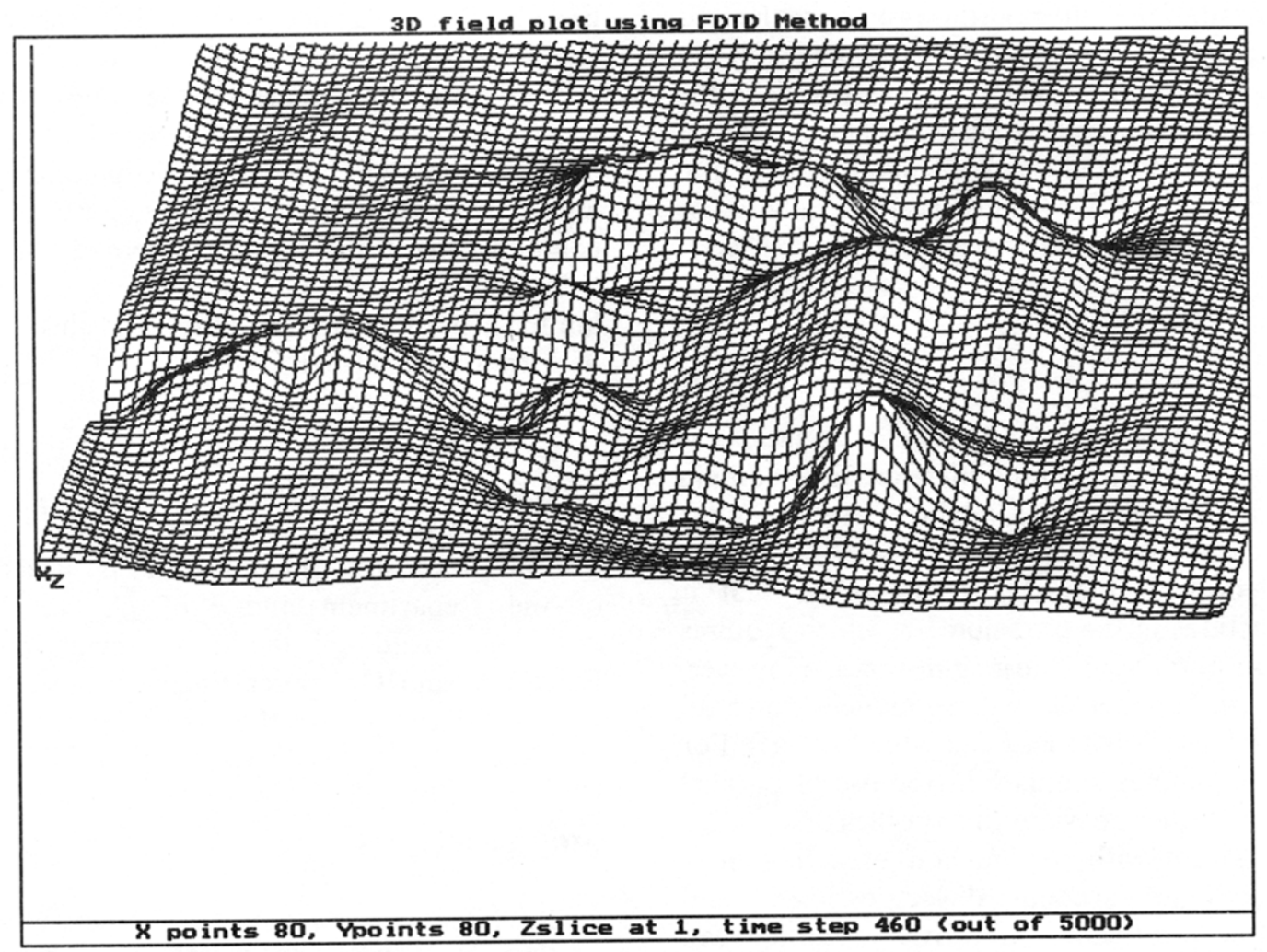

Fig. 19. Simulation after 460 time steps. 


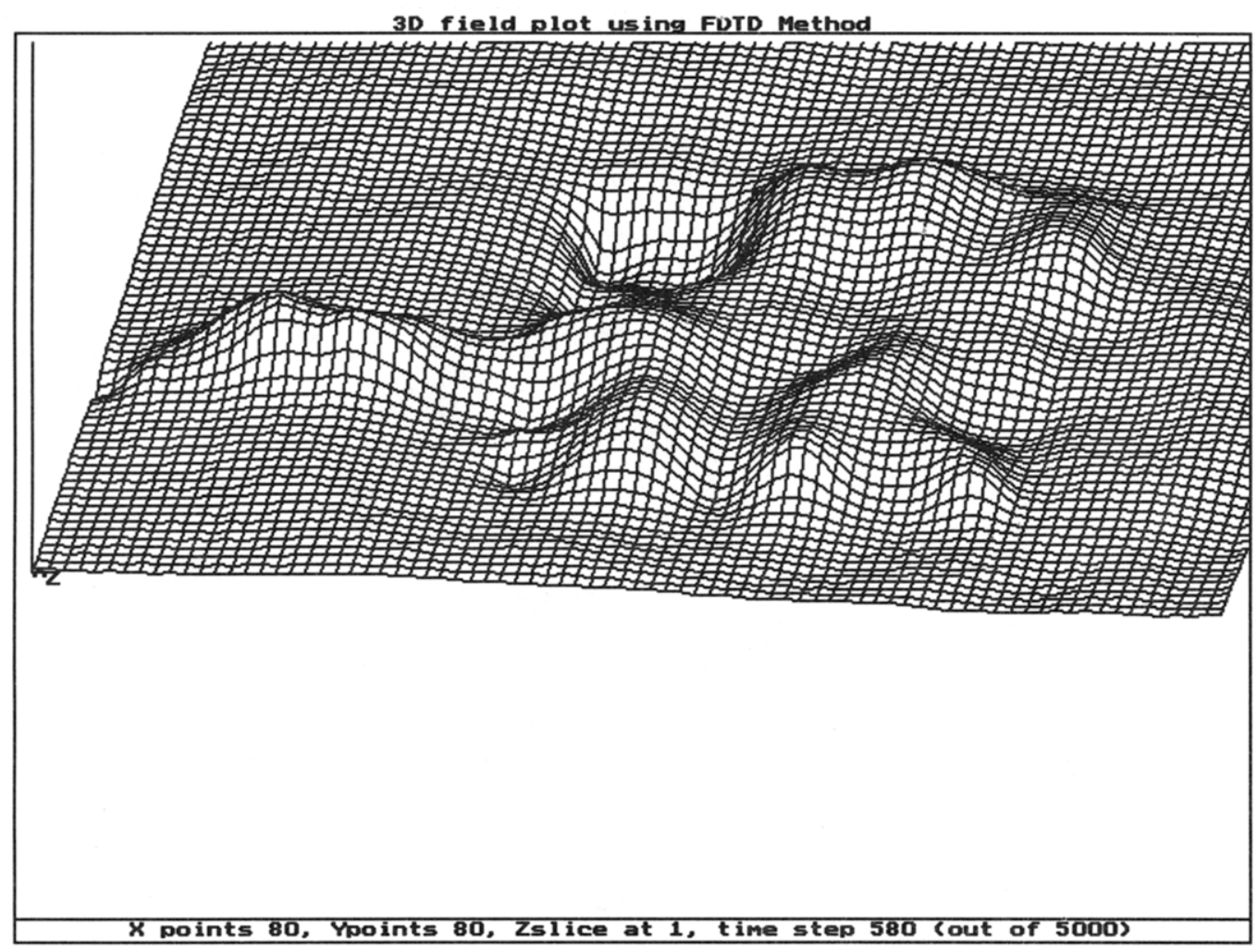

Fig. 20. Simulation after 580 time steps.

is propagating along the feed to the head of the antenna. In Fig. 13, the pulse enters the head of the antenna and a negative pulse is reflected from the interface between the feed and the head of the antenna (this is because the feed has a higher characteristic impedance than the head). As seen in Fig. 14, a negative pulse propagates back to the feed and the pulse entering the head reaches the end of the head and is reflected back. Thus the structure continues to resonate until the pulse is either radiated or absorbed at source or the outer walls.

\section{CONCLUSIONS}

Although the exact solution of Maxwell's equations is complex and tedious, the finite-difference time-domain method is a novel method for solving these equations with great accuracy and simplicity.

One problem with the FDTD method is that it simulates structures in the time domain, which requires large memory storage and large run-times. However, this problem can be reduced by using modern powerful computers (such as $386 / 486$ PCs and workstations). For very large and complex simulations the use of parallel processing will further alleviate this problem.

Another problem with the method used is that it does not take into account dielectric losses and assumes perfect conductors. Improved accuracy can be obtained using sub-gridding methods around discontinuities.
The results obtained clearly show the propagation, reflection and absorption of a Gaussian pulse appropriate to its position in the structure and time. This pulse can be used to determine all required frequency characteristics from DC to the required upper frequency with no change to the model for differernt frequency spectra.

The authors are convinced that parallel processing is an efficient and accurate technique for the simulation of complex structures by the method of FDTD to determine electrical parameters and can be used on limited memory computer systems (such as modern PCs) with transputer arrays.

\section{ACKNOWLEDGEMENTS}

The authors wish to thank Prof. P. H. Beards, the Head of the Department and Prof. A. E. A. Almaini, Associate Head of the Department of Electrical, Electronic and Computer Engineering at Napier University, Edinburgh, for the use of facilities.

\section{BIBLIOGRAPHY}

1. Buchanan, W. J. \& Gupta, N. K. Parallel processing of the three-dimensional finite-difference time-domain method. National Radio Science Colloquium, University of Bradford, 7-8 July 1992. 
2. Buchanan, W. J. \& Gupta, N. K. Simulation of threedimensional finite-difference time-domain method on limited memory systems. IEE Int. Conf. on Computation in Electromagnetics, London, 25-27 November 1992.

3. Hese, J. \& Zutter, D. Modelling of discontinuities in general coaxial waveguide structures by the FDTDmethod. IEEE MTT, 1992, 40 (March).

4. Railton, C. J., Richardson, K. M., McGeehan, J. P. \& Elder, K. F. The prediction of radiation levels from printed circuit boards by means of the FDTD method. IEE Int. Conf. on Computation in Electromagnetics, London, 25-27 November 1992.

5. Sheen, D., Ali, S., Abouzahra, M. \& Kong, J. Application of three-dimensional finite-difference method to the analysis of planar microstrip circuits. IEEE MTT, 1990, 38, 849-57.
6. Shibata, T., Havashi, T. \& Kimura, T. Analysis of microstrip circuits using three-dimensional full-wave electromagnetic field analysis in the time-domain. IEEE MTT, 1988, 36, 1064-70.

7. Svetlana, V., Yee, K. \& Mei, K. A subgridding method for the time-domain finite-difference method to solve Maxwell's equations. IEEE MTT, 1991, 39(3).

8. Taflove, A. The finite-difference time-domain method for electromagnetic scattering and interaction problems. IEEE Trans. Electromagnetic Compatibility, 1980, EMC-22, 191-202.

9. Zang, X., Fang, J. \& Mei, K. Calculations of the dispersive characteristics of microstrips by the FDTD method. IEEE MTT, 1988, 26, 263-7. 